\title{
ZODIACAL DUST BANDS
}

\section{S. F. DERMOTT, D. D. DURDA, B. A. S. GUSTAFSON, S. JAYARAMAN}

\author{
J. C. LIOU AND Y. L. XU \\ Department of Astronomy, \\ University of Florida, \\ Gainesville, FL 32611, U.S.A \\ E-maildermott@astro.ufl.edu
}

\begin{abstract}
One of the outstanding problems in solar system science is the source of the particles that constitute the zodiacal cloud. The zodiacal dust bands discovered by IRAS have a pivotal role in this debate because, without doubt, they are the small, tail end products of asteroidal collisions. Geometrical arguments are probably the strongest and the plane of symmetry of the dust bands places their source firmly in the asteroid belt. A cometary source, Comet Encke for example, could exist at the distance of the mainbelt, but the dynamics of cometary orbits makes the formation of cometary dust bands impossible, unless, of course, there is a significant (comparable in volume to the asteroidal families) source of comets interior to the orbit of Jupiter with low (asteroidal) orbital eccentricities. We have suggested that the dust bands are associated with the prominent asteroidal families. The link with the Themis and Koronis families is good but the link with Eos remains to be proved. We show here by detailed modeling that even though the filtered infrared flux in the $25 \mu \mathrm{m}$ waveband associated with the dust bands is only $\sim 1 \%$ of the total signal, this is only the "tip of the iceberg" and that asteroidal dust associated with the bands constitutes $\sim 10 \%$ of the zodiacal cloud. This result, plus the observed size-frequency distribution of mainbelt asteroids and the observed ratio of the number of family to non-family asteroids allows us to estimate that asteroidal dust accounts for about one third of the zodiacal cloud. The discovery of the "leading-trailing" asymmetry of the zodiacal cloud in the IRAS data and our interpretation of this asymmetry in terms of a ring of asteroidal particles in resonant lock with the Earth is important for two reasons. (1) The existence of the ring strongly suggests that large (diameter $\geq 12 \mu \mathrm{m}$ ) asteroidal particles (or particles with low orbital eccentricities) are transported to the inner solar system by drag forces. (2) The observed ratio of the trailing-leading asymmetry allows an independent estimate of the contribution of asteroidal particles to the zodiacal cloud. These new results have important implications for the source of the interplanetary dust particles (IDPs) collected at the Earth. Because asteroidal particles constitute about one third of the zodiacal cloud and are transported to the inner solar system by drag forces, gravitational focussing by the Earth that results in the preferential capture of particles from orbits with low inclinations and low eccentricities and the possible "funneling" effect of the ring itself, imply that nearly all of the unmelted IDPs collected at the Earth are asteroidal.
\end{abstract}

\section{Introduction}

The primary source of particles in the zodiacal cloud is either asteroidal or cometary, but which of these sources is dominant has proved difficult to determine. The work of the Florida group (Dermott et al., 1984, 1985, 1986, 1988, 1989, $1990,1992,1993,1994)$ has concentrated on modeling the orbital evolution of asteroidal particles. This is partly because of the important role that the IRAS dust bands have in the source debate. It is also true that the asteroidal source is comparatively well-defined and that the small orbital eccentricities of asteroidal particles 
make their dynamics tractable, even in the presence of drag. This is not true for cometary particles. The modeling that we can now do for asteroidal particles cannot, at present, be done for cometary particles. This partly accounts for our lack of work in this area, although some relevant work has recently been completed by Liou (1993). Because of this problem and because asteroids are known to feed the dust bands, the Florida approach is to estimate the contribution of asteroidal particles to the zodiacal cloud. The remainder may primarily be attributed to comets. The cometary trails observed by IRAS are direct evidence of the input of comets to the zodiacal cloud (Sykes and Walker, 1992). Our emphasis on the modeling of an asteroidal source should not be taken to mean that we consider that comets may not have a role in supplying the zodiacal cloud. At present, approximately two thirds of the thermal brightness remains unaccounted for. However, our modeling of the asteroidal dust component is incomplete. The modeling needs to be extended to include (a) a range of particle sizes representing a size distribution and (b) the effects of dust-dust collisions that may increase the effective surface area of the particles as they spiral towards the Sun.

Direct determinations of dust particle orbits using impact detectors or other means are potentially effective ways to discriminate between asteroidal and cometary dust particles. This potential has not yet been realised to the extent of having a major bearing on the question of the origin of the zodiacal cloud. The impact detectors onboard the Galileo and Ulysses spacecraft with effective sensitive areas of $0.1 \mathrm{~m}^{2}$ can detect particles as small as $410^{-15} \mu \mathrm{m}$ at $20 \mathrm{~km} / \mathrm{sec}$ (Grün, this volume). Nearly all the detected dust particles have masses below the $10^{-12} \mathrm{~g}$ mass range, corresponding to diameters in the micron range. This is simply because of the low impact probability of the larger particles. Most of the detected particles correspond to micron or submicron grains that are too small to radiate efficiently in the $25 \mu \mathrm{m}$ waveband. This size range is also thought to be smaller than those particles that contribute most of the zodiacal light in the visual part of the spectrum by the scattering of sunlight (Röser and Staude, 1978).

Grün et al. (1992) point out that the particle trajectories appear to be consistent with an asteroidal source. The orbits are consistent with a population of particles on low inclination $\left(I=0^{\circ}\right.$ to $\left.30^{\circ}\right)$ orbits. Inclination is not affected by radiation pressure, so that the larger particles from the same source would also be expected to be in low inclination orbits. However, the low to moderate eccentricities $(e=$ 0.1 to 0.5 ) are affected by radiation forces and may not be indicative of the orbits of the larger particles.

In Section 2, we estimate the contribution that asteroidal dust associated with the dust bands alone makes to the zodiacal cloud. This leads, in Section 3, to an estimate of the contribution from the asteroid belt as a whole. In Section 4, we present evidence that the Earth is embedded in a solar ring of asteroidal particles (or particles with low orbital eccentricities), strongly suggesting that large asteroidal particles are transported from the mainbelt to the Earth by drag forces. In Section 5, we discuss the implications of these new results for the origin of the IDPs collected at the Earth.

The focus of this paper is the zodiacal dust bands, but our conclusions have implications for the origin of the particles in the zodiacal cloud 'as a whole. Other 
recent reviews on the latter topic that take a much broader viewpoint are by $\mathrm{Di}$ vine (1993), Greenberg (1991), Grün et al. (1985), Leinert and Grün (1990), and Levasseur-Regourd et al. (1991). Recent reviews and papers on dust bands are by Reach $(1991,1992)$ and Sykes et al. (1989).
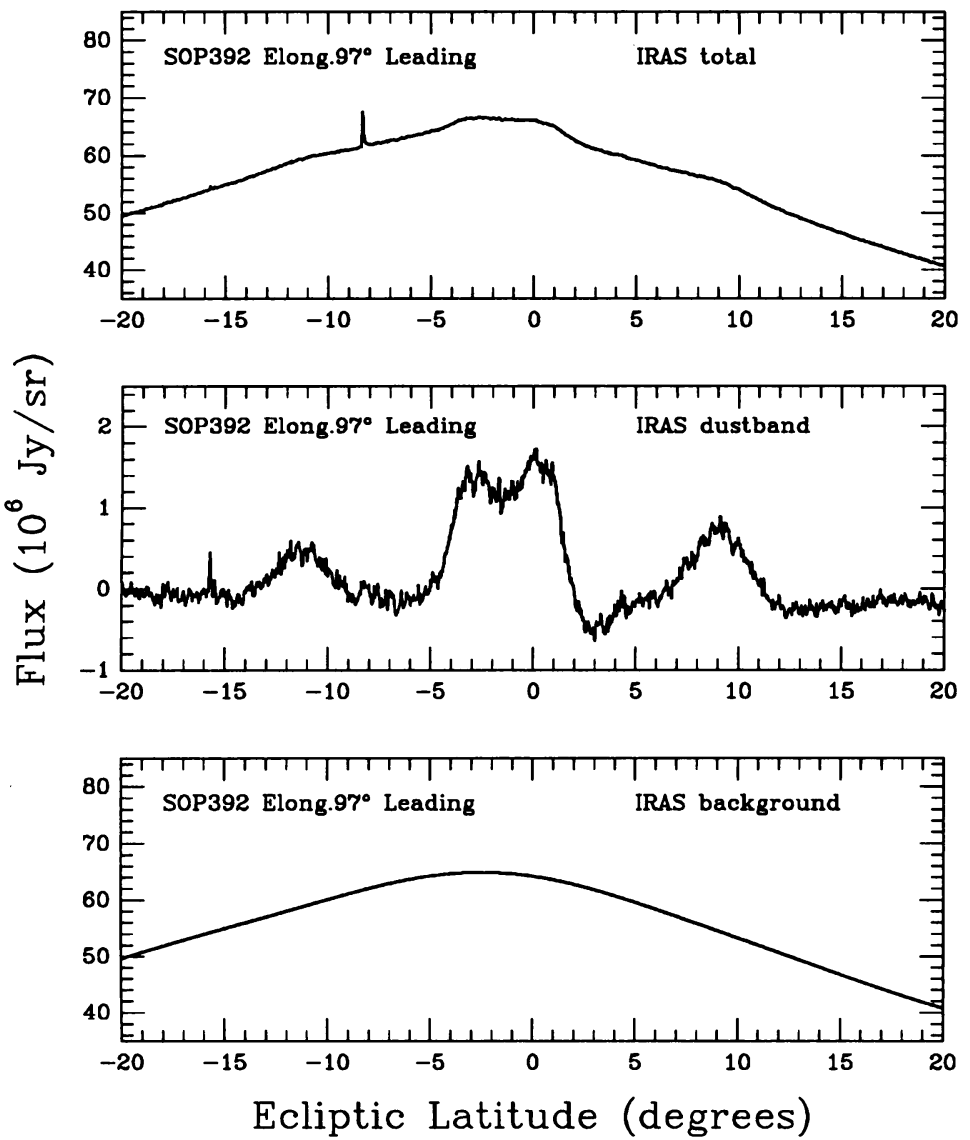

Fig. 1. Top : The total IRAS signal in the $25 \mu m$ waveband. Middle : The narrow scale features (dust bands) subtracted from the total signal using a Fourier filter. Bottom : The broad-scale residual (or background) from the filtering process.

\section{The IRAS Dust Bands}

An example of the data obtained by IRAS in the $25 \mu \mathrm{m}$ waveband (the galactic signal is minimal in this waveband) is shown in Fig.1. This figure shows the variation of thermal flux with ecliptic latitude for an elongation angle of $97^{\circ}$ and for a particular ecliptic longitude of the Earth (SOP 392). Fourier methods are used 
to separate the dust bands from the broad-scale zodiacal background. It needs to be emphasized that the division between short-scale and long-scale features, that must be specified in the filtering process, is entirely arbitrary and yet this divide determines the amplitudes of the dust bands shown in Fig.1. Thus, in Fig.1 the amplitudes of the "ten-degree" bands are $\sim 1 \%$ of the background signal, but these amplitudes could change by a factor as large as $\sim 4$ simply by changing the filter. We show here how this filtering problem can be bypassed, making possible an estimate of the amount of dust associated with the bands. In 1984, we suggested (Dermott et al., 1984) that the dust bands are produced by the gradual comminution of asteroids in the major families. The confirmation of this hypothesis (an ongoing project) has involved : (1) The development of a new secular perturbation theory that includes the effects of Poynting-Robertson light drag, light pressure and solar wind drag on the evolution of the dust particle orbits (Dermott et al., 1992); (2) The production of a new high resolution Zodiacal History File by IPAC (the Infrared Processing and Analysis Center at Caltech); (3) The development of the SIMUL code : a three-dimensional numerical model that allows the calculation of the thermal flux produced by any particular distribution of dust particle orbits (Dermott et al., 1988). These tools allow the modeling of the asteroidal component of the zodiacal cloud from source to sink.

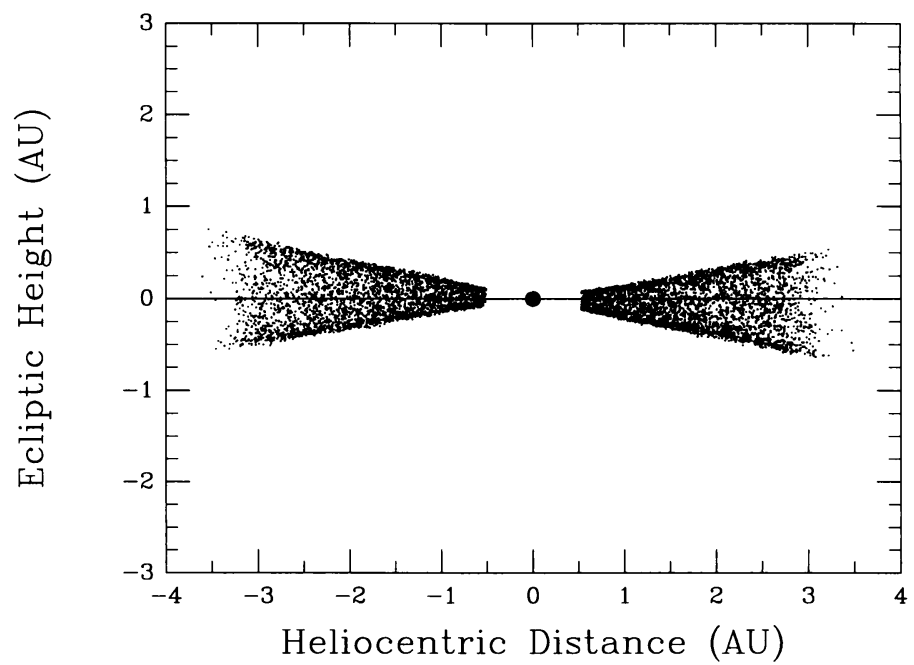

Fig. 2. Vertical slice through a model dust band based on numerical integration of the positions of $9 \mu m$ diameter asteroidal particles derived from the Eos family. The integration was stopped at 0.5 A.U.

The cross-sectional structure of a typical dust band is shown in Fig.2. This figure shows the positions of $9 \mu m$ diameter particles originating from the Eos family. The particles spiral towards the Sun due to the action of drag forces. During this orbital evolution there are small (calculable) changes in the forced inclinations and nodes. 
These forced elements define the effective plane of symmetry of the cloud and vary with particle size and with distance from the Sun. However, the proper inclination remains constant (Dermott et al., 1992) and it is this property that gives rise to an observable pair of dustbands. Because the proper inclination remains constant, there is an enhancement in particle number density at the upper and lower surfaces of the band and this gives rise to an enhancement in flux when the dust band is viewed in a direction close to one of these surfaces (see fig. 2). A pair of dust bands is observed because only the peaks in the flux that rise significantly above that of the broadscale background are observed. Most of the flux arising from the dust associated with the band merges into the background.

The orbital evolution of cometary particles is quite different (Liou, 1993). Because of their higher eccentricities, the variations in the inclinations are not decoupled from those of the eccentricities and the inclination with respect to the plane associated with the forced inclination is no longer constant. Drag forces acting on particles released from Comet Encke with initial inclinations with respect to the forced plane of, for example, 10 degrees experience dispersion and increases up to values as large as 40 degrees (Liou, 1993). This distribution of inclinations may have a role in determining the shape of the background cloud, but it is impossible for such a widely dispersed distribution of inclinations to produce an observable pair of dust bands.

The location of the source of the dust bands can be determined from their observed plane of symmetry. The latitudes of the peaks shown in the middle panel of fig. 1 are closely associated with those particles in the more distant (from the Sun) regions of the dust band. The variation with the longitude of the Earth of the latitudes of these peaks, observed at a constant elongation angle of $90^{\circ}$ in the trailing (that is, opposite to the Earth's orbital motion) and in the leading directions, can be used to determine the orientation of the plane of symmetry. Highly precise values of these latitudes can be obtained by exploiting the IRAS observing sequence (Dermott et al. 1993, 1994). To obtain good all-sky coverage, the elongation angle used by IRAS was not held constant at $90^{\circ}$, but was shifted systematically from scan to scan over periods of a few weeks to ensure complete coverage of each "lune" of the sky. For the first two thirds of the mission, the elongation angle, while being kept within the range $\sim 80^{\circ}$ to $\sim 100^{\circ}$, was systematically increased from scan to scan for scans in the trailing direction, while for scans in the leading direction the elongation was systematically decreased. These regularities allow us to increase the signal to noise ratio by using all the data in a given lune to obtain (a) the average latitude of a dust band peak for an elongation angle of $90^{\circ}$ and (b) the ecliptic longitude of the Earth corresponding to that elongation angle (Dermott et al. 1993, 1994). By plotting these latitudes as a function of the longitude of the Earth, we can find the nodes of the band with respect to the ecliptic. These are the ecliptic longitudes of the Earth at which the latitudes of the mid-points between the North and South peaks in the leading and trailing directions are equal and opposite and equal in magnitude to the forced inclination. Results from Dermott et al. (1992, 1993) are shown in Table 1. The observed plane of symmetry of the "ten-degree" bands is quite distinct from that of the background cloud, but is closely similar to that of the asteroidal families (Eos and Themis, for example) in the outer part of 
the mainbelt. Other work on the orientation of the zodiacal cloud includes that by Hauser (1988), and Leinert et al. (1980).

Table 1: Orientations of planes of symmetry with respect to Ecliptic.

\begin{tabular}{lccccc}
\hline \multicolumn{1}{c}{ Feature } & Waveband & \multicolumn{2}{c}{ Ascending } & \multicolumn{2}{c}{ Descending } \\
& & Inclination & Node & Inclination & Node \\
\hline 10 degree bands & $25 \mu m$ & $1.16 \pm 0.09^{\circ}$ & $99.7 \pm 7.8^{\circ}$ & $1.08 \pm 0.06^{\circ}$ & $265.5 \pm 7.8^{\circ}$ \\
Eos Family & & $1.19^{\circ}$ & $97.1^{\circ}$ & $1.19^{\circ}$ & $277.1^{\circ}$ \\
Themis Family & & $1.22^{\circ}$ & $97.8^{\circ}$ & $1.22^{\circ}$ & $277.8^{\circ}$ \\
Zodiacal Cloud & $25 \mu m$ & $1.60 \pm 0.02^{\circ}$ & $47.2 \pm 0.8^{\circ}$ & $1.52 \pm 0.02^{\circ}$ & $230.7 \pm 0.8^{\circ}$ \\
\hline
\end{tabular}

An illustrative example of the method that we use to bypass the filtering problem and to estimate the fraction of the zodiacal cloud that is associated with the dust bands is outlined in fig. 3 shown on the next page. This particular figure shows the results of the modeling that we have done to account for the shape of the dust band shown in fig. 1. Previous work on the modeling of the background zodiacal cloud (Dermott et al. 1992) has shown that the plane of symmetry of the cloud can be accounted for by, for example, asteroidal particles with diameters of $12 \mu \mathrm{m}$ and densities of $2.7 \mathrm{gm} / \mathrm{cm}^{3}$ that spiral in from the mainbelt to the Sun without disruption. A more complete model would employ a range of particles sizes and consider the effects of particle breakup due to dust-dust collisions, but our modeling is not yet that sophisticated. In this paper, we use the same single-size asteroidal particles to model the orbital evolution of dust associated with the asteroid families.

The model profile shown in fig. 3a(i) was produced using dust from six prominent families : Themis, Koronis, Eos, Nysa, Dora and Gefion as defined by Zappalà et al. $(1990,1994)$. The area of dust associated with each family was adjusted to match the observed profile shown in the middle panel of fig. 1 using the following iterative procedure. The raw profile shown in fig. $3 a(i)$ was added to the background profile shown in the bottom panel of fig. 1 . The resultant sum was then passed through the same filter that was used to produce the observed dust band. This results in a model dust band that can be compared with the observations - see fig. $3 b(i)$. However, most of the flux seen in fig. $3 a(i)$ goes into the background signal and hence the filtered background exceeds the observed background - see fig. $3 \mathrm{~b}$ (ii). This excess is subtracted from the background shown in the bottom panel of fig. 1. before adding the flux shown in fig. $3 a(i)$. On filtering the resultant sum, we now obtain a model dust band profile as well as a background residual that are the same as those shown in fig. 1 - see fig. 3c. We can show that these results are largely independent of the filter used. Similar modeling has been repeated for many other dust band profiles spanning a wide range of elongation angles (Dermott et al. 1992). Thus, by using the same filter in the modeling process that we use to define the observed dust bands, and iterating, we are able to bypass the arbitrary 

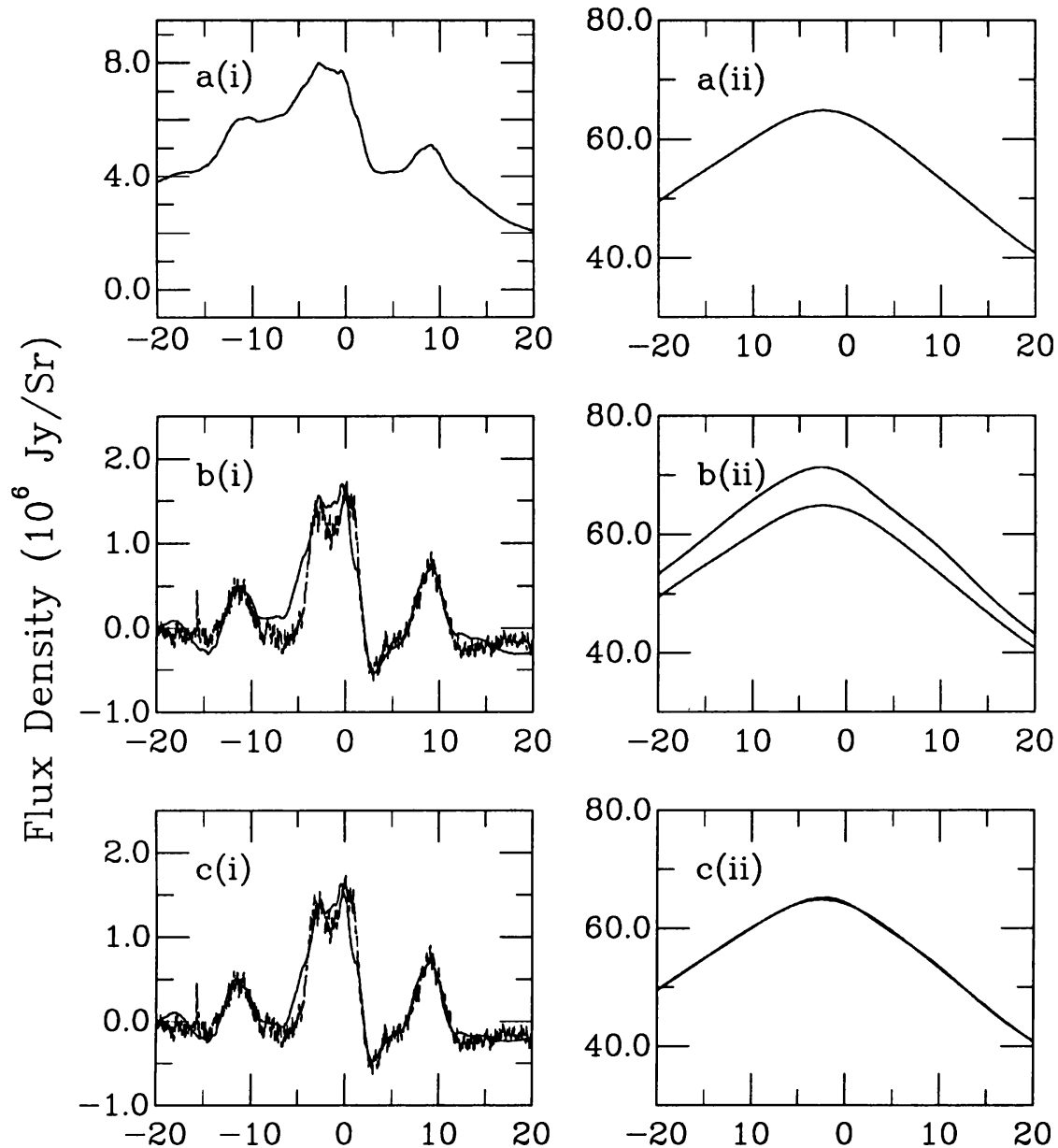

Ecliptic Latitude

Fig. 3. Outline of the filtering process : (a) Top panel shows the raw model dustband (i) and the observed broadscale back ground (ii); (b) In the first iteration we add a(i) and a(ii) and filter to obtain a model dustband (smooth curve in (i)) similar to observations (noisy curve in (i)) and a background (upper curve in (ii)) which is higher than the observed background (lower curve in (ii)); (c) In the final iteration we subtract the excess flux shown in b(ii) from the background in a(ii) and add a(i) before filtering to obtain the final dustband shown in $c(i)$ and the final background shown in c(ii) that agree with the observations. 
divide associated with the filter. This allows us to estimate the true extent of the underlying dust band. Given that the peak flux shown in fig. $3 a(i)$ is about $10 \%$ of that shown in fig. $3 \mathrm{a}$ (ii), we deduce that about $10 \%$ of the dust in the zodiacal cloud is associated with the dust bands and hence with the prominent Zappala et al. $(1990,1994)$ asteroid families. We do need to assume that the dust derives from a number of families and not just the two largest (Eos and Themis). In fact, we are not confident that Eos is the dominant contributor to the ten-degree bands, but this will not be certain until we have a more sophisticated model that uses a realistic range of particle sizes. However, these further considerations may change the amount of dust that we associate with particular families but they are unlikely to effect significantly our estimate of the total contribution of the families as a whole to the zodiacal cloud.

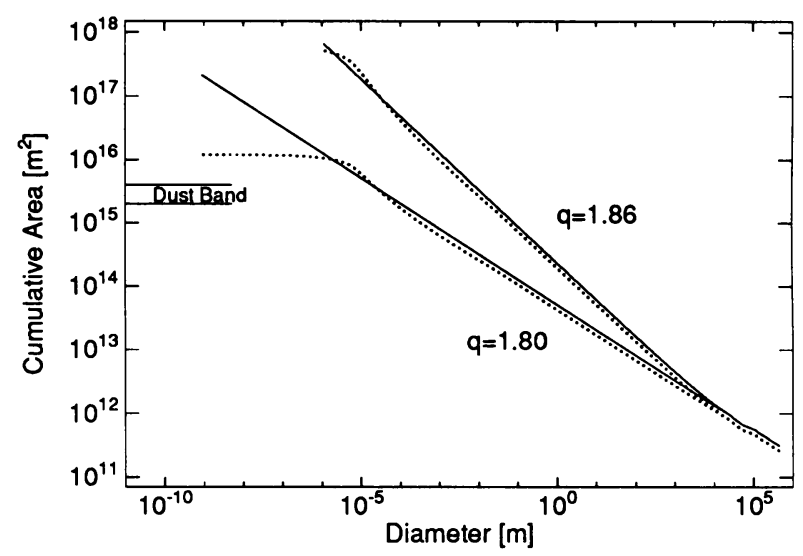

Fig. 4. Cumulative area of dust associated with the Zappalà et al. $(1990,1994)$ families asteroid families for two values of the size-frequency index $q$. The solid lines represent the geometrical area, while the dotted lines are the effective area calculated for astronomical silicate using Mie theory. The cumulative area of dust needed to account for the IRAS observations of the dustbands is represented by the banded region on the left.

\section{Asteroidal Collisions}

The collisional evolution of the asteroid belt has recently been investigated by Durda (1993). The result of Dohnanyi (1969), that the size-frequency index $q$ is 1.833 holds, but only if the strength of the asteroids is independent of their size. Our lack of knowledge of how strength actually depends on size across the micron to $100 \mathrm{~km}$ size range (11 decades!) makes the relationship between the total volume of asteroids in collisional "equilibrium" that feed the zodiacal cloud (those asteroids 


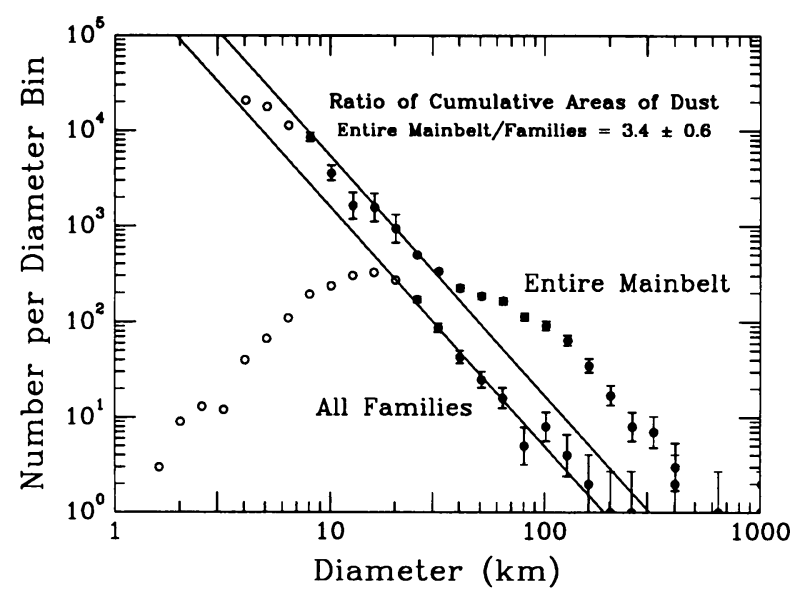

Fig. 5. Size-frequency distribution of (a) all asteroids in the mainbelt (upper line), and (b) the asteroids in the Zappalà et al. $(1990,1994)$ families (lower line).

with diameters $\leq 30 \mathrm{~km}$ ) and the effective area of dust that emits radiation in a given waveband somewhat tentative. Thus, even though asteroids are a comparatively well-defined source, we cannot obtain a useful estimate of the effective area of asteroidal dust from first principles. The situation with estimating the contribution of comets to the zodiacal cloud is even worse. Our knowledge of the size-frequency distribution of comets is minimal to non-existent and estimates of the area of dust associated with this source are of little value. In fig. 4 , we relate the total volume in all the Zappalà et al. $(1990,1994)$ asteroid families (Durda, 1993) to the associated effective area of dust in the IRAS $25 \mu \mathrm{m}$ waveband using two assumed values for $q$ that span the canonical value of 1.833 . It would appear that the predicted area of dust may be more than adequate to account for the observations. However, there are many uncertainties. One interesting fact discovered by Durda (1993) is that the value of $q$ may vary from family to family and may be different from that of the general background (the stronger the asteroidal grains, the greater the amount of dust that will be associated with a given source volume). These uncertainties make predictions even more difficult. However, we can use the dust band observations to make a moderately robust estimate of the contribution of the asteroid belt as a whole to the zodiacal cloud. Given that the dust bands are related to the Zappalà et al. $(1990,1994)$ families, and that the dust associated with these families constitutes $10 \%$ of the zodiacal cloud, then the observation that the ratio of family to non-family asteroids in the collisionally evolved asteroidal population is approximately $3.4: 1$ implies that the asteroid belt as a whole is the source of $\sim 1 / 3$ of the dust in the zodiacal cloud (see fig. 5 ). Due to the stochastic nature of collisions in the asteroid belt and our lack of knowledge of the number of small, collisionally-evolved asteroids in the outer regions of the asteroid belt, the latter 
estimate may be uncertain by a factor of two or more (Durda, 1993).

\section{The Earth's Asteroidal Ring}

The IRAS spacecraft has given us our highest resolution data on the structure of the zodiacal cloud. Dermott et al. (1988) analysed this data and pointed out a marked but peculiar asymmetry, namely that observations of the peak brightness of the zodiacal cloud in the trailing direction (that is, opposite to the Earth's orbital motion) are consistently greater than those in the leading direction. This finding was later confirmed by Reach (1991).

Collisions amongst the asteroids result in the grinding down of the asteroid belt and the production of small dust particles that spiral towards to the Sun due to the action of drag forces. Numerical integrations show that a significant fraction of these particles are temporarily trapped in corotational resonances external to the orbits of each of the terrestrial planets, particularly the Earth (Jackson and Zook, 1989; Dermott et al., 1994) - Figs.6-8. The result is that the Earth is embedded in a ring of asteroidal particles (or, more correctly, a ring of particles with low orbital eccenticities) that is longitudinally near uniform apart from a cavity that contains the planet. The action of drag introduces a phase lag into the equation of motion of these resonant particles. The physical manifestation of this is a phase difference between the longitude of conjunction of the dust particle and the Earth and the apocenter of the dust particle's orbit, with the result that the position of the Earth within the cavity is markedly asymmetric, with the Earth closer to the edge of the cavity in the trailing orbital direction (opposite to the Earth's orbital motion) than in the leading orbital direction (fig. 8). The above discussion certainly applies to mainbelt asteroidal particles. The higher orbital eccentricities of most cometary particles in the vicinity of the Earth (Jackson and Zook, 1992) and of particles deriving from the disruption of near-Earth asteroids make resonant trapping of these particles considerably less likely. We do not deny that some cometary particles in the vicinity of the Earth may have very low orbital eccentricities, but we consider it very unlikely that cometary particles in these orbits constitute a source comparable to that of mainbelt asteroids.

The size-frequency distribution of asteroidal particles is such that the effective area of dust and hence the thermal flux from the ring observed in a given IRAS waveband is dominated by flux from the smaller (but not the smallest) particles in the distribution. These particles have high phase shifts with the result that the dynamics of resonance is in the non-adiabatic regime, for which analytic theory is only a guide and reliable results must be obtained by numerical integration. The drag rates on very small particles can be too high to allow resonance trapping. To obtain a detailed description of the structure of the ring, the dynamics of a wide range of particle sizes need to be considered. Dermott et al. (1994) estimated that asteroidal particles of diameter $12 \mu \mathrm{m}$ and density $2.7 \mathrm{~g} / \mathrm{cm}^{3}$ are close to the critical size for trapping into the resonances in significant numbers and estimated the structure of the ring from the dynamics of those particles alone.

On integrating the orbits of 2048 particles originating from the main asteroid belt, we found that up to $20 \%$ of the particles are trapped in first-order resonances 

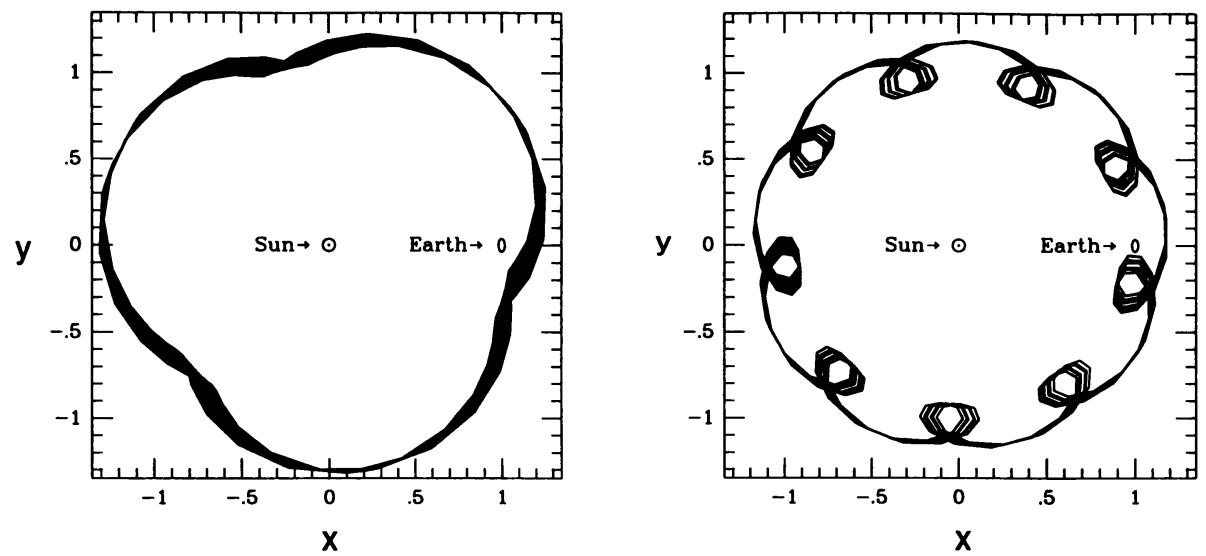

Fig. 6. Orbits of $12 \mu m$ asteroidal particles in (a) $3: 4$ resonance and (b) $9: 10$ resonance tracked over one complete libration period and plotted in a frame centred on the sun and rotating with the Earth's mean motion.

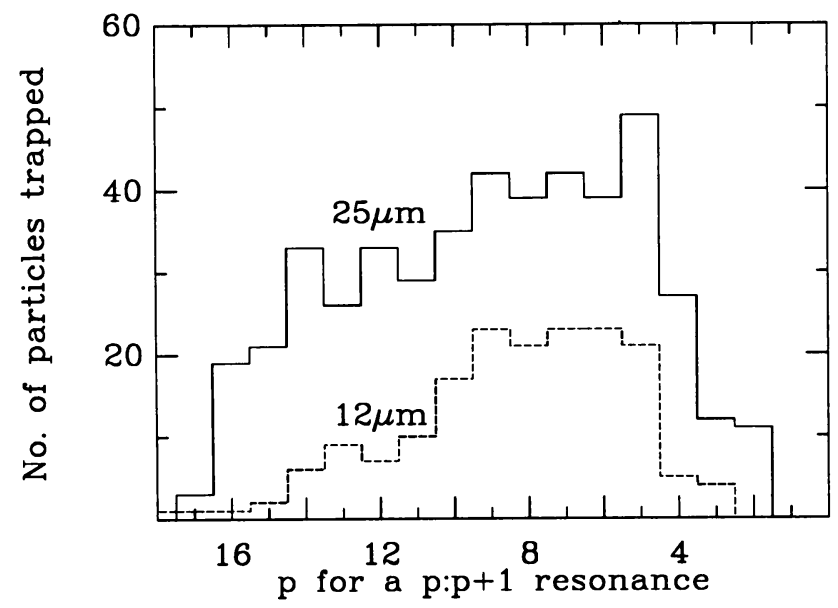

Fig. 7. Relative probabilities of capture into different resonances of asteroidal particles of diameter $12 \mu m$ and $25 \mu m$.

with $p: p+1$ (the ratio of mean motions) ranging from $3: 4$ to $17: 18$ with occasionally even higher values of p. fig. 7 shows the relative probability of capture into the individual resonances for two sizes of particles.The average 
trapping time for particles in these resonances was found empirically. Our final model is a simulated 'image' binned in pixels of $0.04 \times 0.04$ A.U. (fig. 8, left). The resultant image obtained by superposition of the various paths weighted according to the probability of capture and the trapping times shows that, in a rotating reference frame, the trapped particles form a near-uniform ring around the Sun that corotates in inertial space with the Earth.

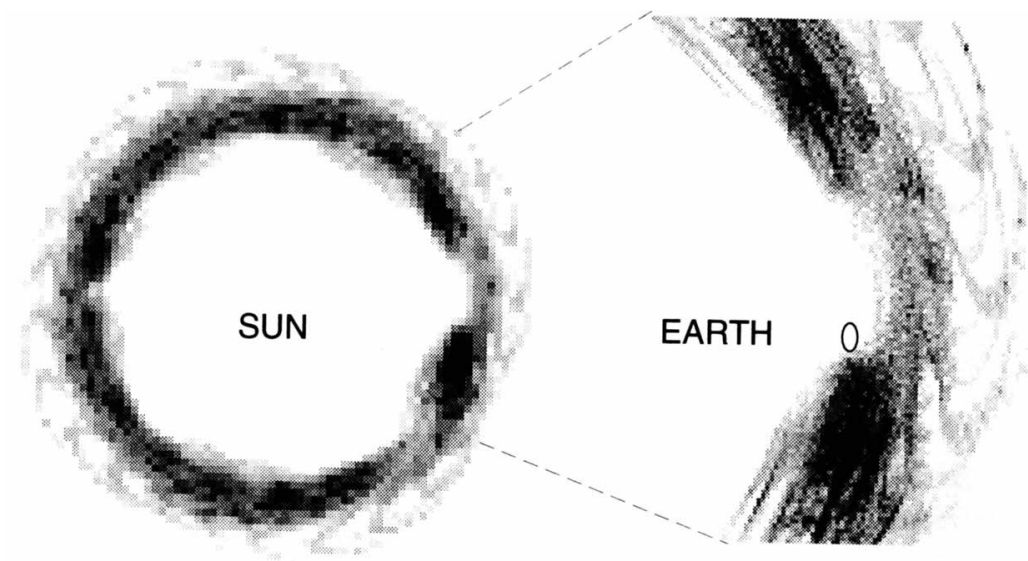

Fig. 8. Numerically simulated images of a heliocentric ring of asteroidal particles corotating with the Earth shown in low resolution on the left and high resolution on the right. The path of the Earth is a small $2: 1$ ellipse that resides in a cavity in the ring.

The peripheral arc-like structures in the ring are artifacts caused by the limited number of single-size particles considered in the model. However, the other noticeable features in fig. 8 are real. Firstly, the existence of a cavity in the ring at the location of the Earth. This is seen more clearly using a higher resolution of $0.01 \mathrm{x}$ 0.01 A.U. The 2 : 1 ellipse in fig. 8 (on the right) is the epicycle of the Earth's orbit in the rotating reference frame. Secondly, fig. 8 also shows that apart from an asymmetry in the mean position of the Earth in the ring's cavity, there is also a marked asymmetry in the longitudinal variation of the particle number density. The increase in resonance strength and the corresponding decrease in phase shift with increasing $p$ value, disperses the longitudes of the pericentres of the particle paths in the rotating frame in the leading direction while concentrating the longitudes of the pericenters in the trailing direction with the result that there is marked enhancement in particle number density behind the Earth in its orbit, as if the Earth had a trailing cloud of dust permanently in its wake. We consider that these asymmetries act together to produce the observed trailing/leading flux ratios of the zodiacal cloud.

We analysed all the IRAS observations of the zodiacal cloud and showed that when viewed at a constant elongation angle of $90^{\circ}$, the ecliptic brightness of the cloud is systematically greater by $\sim 3 \%$ in the trailing direction than in the leading 


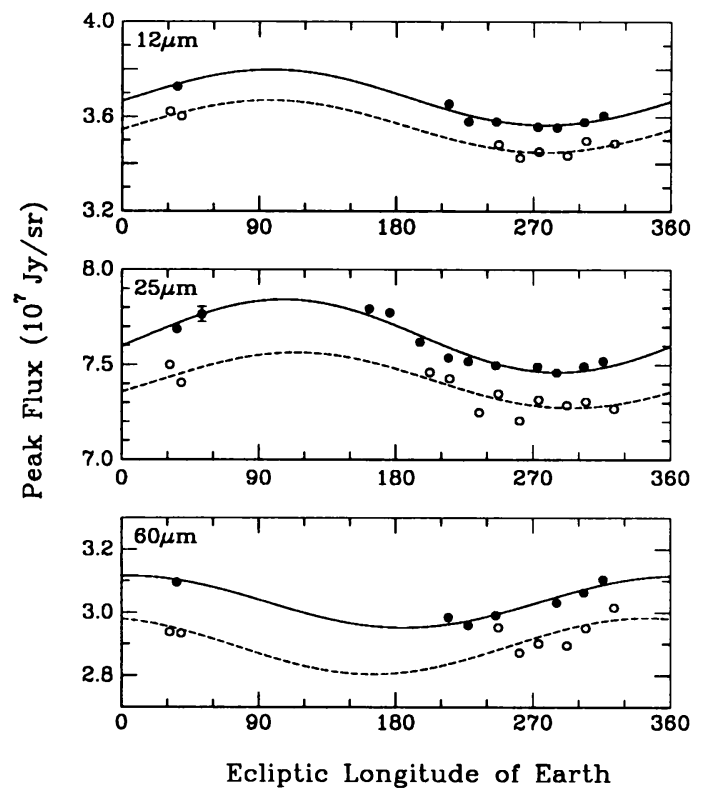

Fig. 9. Variation with the ecliptic longitude of Earth of the peak brightness of the zodiacal cloud as observed by IRAS in the three infrared wavebands at an elongation of $90^{\circ}$ in the trailing direction (filled circles and upper curve) and in the leading direction (open circles and lower curve).

direction. This asymmetry is observed at all times of the year and in all three of the IRAS wavebands in which the zodiacal cloud is clearly observed (fig. 9). The existence of asteroidal families and their associated dust bands (also discovered by IRAS) are evidence that the asteroid belt is a significant source of dust in the zodiacal cloud. These new observations will give quantitative information both on the rate of transport of asteroidal dust to the Earth and on the rate of grinding down of the asteroid belt.

We have also calculated that if the zodiacal cloud consisted of $12 \mu m$ diameter asteroidal particles alone, with number densities sufficient to account for the observed peak brightness in the IRAS $25 \mu m$ waveband, then the trailing/leading asymmetry would be three times higher than that observed. There are two reasons why this must be an overestimate. Small asteroidal particles that exist in the background cloud that cannot be trapped in the resonances because of their high drag rates will act to reduce the trailing/leading asymmetry, as will any cometary particles with comparatively high orbital eccentricities that contribute to the background flux. These calculations and the observed trailing/leading flux ratios imply that, in terms of effective area, the fraction of asteroidal particles that can contribute to the ring is about one third of the total asteroidal particles in the cloud, but more sophisticated calculations are needed here, and more work needs to be done on the 
distinction of the orbits of cometary and asteroidal particles in the vicinity of the Earth.

\section{Source of IDPs collected at the Earth}

Without resonant trapping most dust particles spiral past the Earth without striking the planet. However, most particles trapped in resonances are released from these resonances due to close encounters with the Earth. Thus, the ring may act as a funnel through which asteroidal particles are deposited in the Earth's atmosphere. This statement needs to be verified, but could have important implications for the efficiency of the transport of carbonaceous material from the asteroid belt to the Earth.

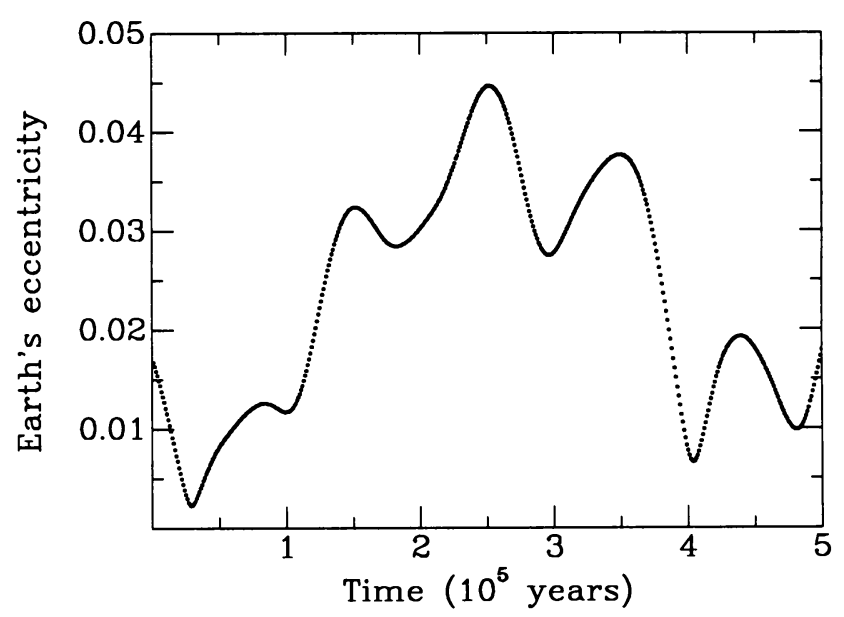

Fig. 10. Secular variation of the Earth's orbital eccentricity

Flynn (1989), following Opik (1951), has recently pointed out that the low geocentric velocities of asteroidal particles result in an increase in the gravitational cross-section of the Earth by a factor of $1+v_{e}^{2} / v_{g}^{2}$ due to gravitational focussing, where $v_{e}$ is the Earth's escape velocity $(11.2 \mathrm{~km} / \mathrm{s})$ and $v_{g}$ is the geocentric velocity of the particle. Because asteroidal particles probably constitute one third of the zodiacal cloud and are transported to the inner solar system by drag forces, gravitational focussing by the Earth that results in the preferential capture of particles from orbits with low inclinations and low eccentricities and the possible "funneling" effect of the ring itself, imply that nearly all of the unmelted IDPs collected at the Earth are probably asteroidal. If this is the case, then there will be marked long-term variations in the magnitude of the Earth's resonant ring and in the deposition rate of asteroidal particles on the Earth due to the secular variation of the Earth's orbital eccentricity (fig. 10) and the corresponding variations in the forced eccentricities of the particle orbits. These periodicities should be searched for in 
Antarctic ice cores. There will also be annual variations in the deposition rate of asteroidal particles on the Earth due to the Earth's orbital eccentricity that determines the distance of the Earth from the edge of ring cavity (right panel of fig. 8) and also due to the effect of forced inclinations on orbits of low proper inclination (Dermott and Liou, 1994). Strong indications of annual variations exist in the radio observations of meteor echoes (Suzuki, 1991).

\section{Acknowledgements}

This work was supported by NASA grants NAGW 1923 and NAG 9-440, by the University of Florida and the IBM Corporation through their Research Computing Initiative at the Northeast Regional Data Center, and by the Geophysics Directorate of the United States Air Force Phillips Laboratory through subcontracts with Mission Research Corporation and General Research Corporation under the Celestial Background Scene Descriptor program.

\section{References}

Dermott, S.F., Nicholson, P.D., Burns, J.A., and Houck, J.R. : 1984, "Origin of solar system dustbands discovered by IRAS". Nature, 312, 505-509.

Dermott, S.F., Nicholson, P.D., Burns, J.A., and Houck, J.R. : 1985, "An analysis of the Solar System Dust Bands". In Properties and Interaction of Interplanetary Dust (R.H. Giese, P. Lamy, Eds.), 395-409, Reidel, Dordrecht.

Dermott, S.F., Nicholson, P.D., and Wolven, B. : 1986, "Preliminary Analysis of the IRAS Solar System Dust Bands". In Asteroids Comets and Meteors II (C-I. Lagerkvist B.A. Lindblad, H. Lundstedt, H. Rickman, Eds.), 583-594, Uppsala Universitet Reprocentralen.

Dermott, S.F., Nicholson, P.D., Kim, Y., Wolven, B., and Tedesco, E.F. : 1988, "The impact of IRAS on Asteroidal Science". In Comets to Cosmology (A. Lawrence, Ed.), 3-18, Springer-Verlag, Berlin.

Dermott, S.F., and Nicholson, P.D. : 1989, "IRAS Dustbands and the origin of the Zodiacal Cloud". Highlights of Astronomy, 8, 259-266.

Dermott, S.F., Nicholson, P.D., Gomes, R.S. and Malhotra, R. : 1990 , "Modelling the IRAS solar system dustbands". Adv. Space Res., 10, 165-172.

Dermott, S. F., et al : 1992 : "Dynamics of the Zodiacal Cloud". In Chaos, Resonance, and Collective Dynamical Phenomena in the Solar System (S. Ferraz-Mello,Ed.), 333347, Kluwer, Dordrecht.

Dermott, S. F., et al. : 1993, "The Origin of IRAS Dustbands". In Meteroids and Their Parent Bodies (J. Stohl, I.P. Williams Eds.), 357-366, Astronomical Inst., Slovak. Acad. Sci., Bratislava.

Dermott, S.F., Jayaraman, S., Xu, Y.L. and Liou, J.C. : 1994, "IRAS observations of a ring around the sun : Asteroidal particles in resonant lock with the Earth". Nature, (submitted).

Deul, E.R., and Walker, H.J. : 1989, "Responsivity variations in the IRAS survey". Astron. Astrophys. Suppl. Ser., 81, 207-214.

Divine, N. : 1993, "Five Populations of Interplanetary Meteoroids". J. Geophys. Res., 98, 17029-17048.

Dohnayi, J.S. : 1969, "Collisional model of asteroids and their debris". J. Geophys. Res., 74, 2531-2554.

Durda, D.D. : 1993, Ph.D. Thesis, University of Florida. 
Flynn, G.J. : 1989, "Atmospheric entry heating : A criterion to distinguish between asteroidal and planetary sources of dust". Icarus, 77, 287-310.

Greenberg, J. M. : 1991, "The Interplanetary medium is thriving". In Origin and Evolution of Interplanetary Dust (A. C. Levasseur-Regourd, H. Hasegawa Eds.), 443-451, Kluwer, Dordrecht.

Grün, E., Zook, H. A., Fechtig, H., and Giese, R.H. : 1985, "Collisional balance of the meteoritic complex". Icarus, 62, 244-272.

Grün, E., Fechtig, H. and Kissel, J. : 1985, "Orbits of Interplanteray dust particles insider $1 \mathrm{AU}$ as observed by Helios". In Properties and Interactions of Interplanetary dust (R. Giese, P. Lamy, Eds.), 101-111, Reidel, Dordrecht.

Grün, E., et al : 1992, "Galileo and Ulysses dust measurements : From Venus to Jupiter". Geophys. Res. Lett., 19, 1311-1314.

Hauser, M. G. : 1988, "Models for infrared emission from Zodiacal Dust". In Comets to Cosmology (A. Lawrence Ed.), 27-39, Springer-Verlag, Berlin.

Jackson, A.A. and Zook, H.A.: 1989, "A Solar System dust ring with the Earth as its shepherd". Nature, 337, 629-631.

Jackson, A.A. and Zook, H.A. : 1992, "Orbital Evolution of Dust particles from Comets and Asteroids". Icarus, 97, 70-84.

Leinert, C., Hanner, M., Ritcher and I., Pitz, E. : 1980, "The plane of symmetry of the interplanetary dust in the inner solar system". Astron. Astrophys., 82, 328-336.

Leinert, C. and Grũn, E. : 1990, "Interplanetary Dust". In Physics of the Inner Heliosphere I (R. Schwenn, E. Marsch, Eds.) 207-275, Springer-Verlag, Heidelberg.

Levasseur-Regourd, A.C., Renard, J.B. and Dumont, R. : 1991, "The Zodiacal cloud complex". In Origin and Evolution of Interplanetary Dust (A.C. Levasseur-Regourd, H. Hasegawa, Eds.), 131-138, Kluwer, Dordrecht.

Liou, J.C. : 1993, Ph.D. Thesis, University of Florida.

Low. F.J., et al. : 1984, "Infrared cirrcus : New components of the extended infrared emission". Astrophys. J. Lett. 278, L19-L22.

Opik, E.J. : 1951, "Collision probabilities with the planets and the distribution of interplanetary dust". In Proc. Roy. Irish Acad., 54, Sect A, 165-199.

Reach, W.T. : 1991, "Zodiacal Emission II. Dust near ecliptic". Astrophys. J., 369, 529543.

Reach, W.T. : 1992, "Zodiacal Emission III. Dust near Asteroid Belt". Astrophys. J., 392, 289-299.

Röser, S. and Staude, H.J. : 1978, "The zodiacal light from 1500 Åto 60 micron". Astron. Astrophys., 67, 381-394.

Suzuki, K. : 1991, "The annual variation of radio meteor echoes observed from 1981 to 1985". In Origin and Evolution of Interplanetary Dust (A.C. Levasseur-Regourd, H. Hasegawa, Eds.), 319-322, Kluwer, Dordrecht.

Sykes, M.V., Greenberg, R., Dermott, S.F., Nicholson, P.D., Burns, J.A. and Gautier, T.N. : 1989, "Dust Bands in the Asteroid Belt". In Asteroids II (R. Binzel, T. Gehrels, M.S. Matthews, Eds), 336-367, Univ. of Arizona Press., Tucson.

Sykes, M.V. and Walker, R.G : 1992, "Cometary Dust Trails". Icarus, 95, 180-200.

Zappalà, V., Cellino, A. Farinella, P. and Knežević, Z. : 1990, "Asteroid Families I. Identification by hierarchial clustering and reliability assessment". Astron. J., 100, 2030-2046.

Zappalà, V., Cellino, A. Farinella, P. and Milani, A. : 1994, "Asteroid Families II. Extended emission to unnumbered multiopposition asteroids". Astron. J., 107, 772-801. 